\title{
GAD-alum immunotherapy in type 1 diabetes expands bifunctional Th1/Th2 autoreactive CD4 T cells
}

\author{
Sefina Arif ${ }^{1}$ - Iria Gomez-Tourino ${ }^{1} \cdot$ Yogesh Kamra $^{1}$ • Irma Pujol-Autonell ${ }^{1} \cdot$ Emily Hanton $^{1} \cdot$ Timothy Tree $^{1}$. \\ Daisy Melandri ${ }^{1} \cdot$ Caroline Hull $^{1}$ - Diane K. Wherrett ${ }^{2} \cdot$ Craig Beam $^{3}$ - Bart O. Roep ${ }^{4} \cdot$ Anna Lorenc $^{1}$. \\ Mark Peakman $^{1,5}$ (D)
}

Received: 22 January 2020 / Accepted: 18 February 2020/Published online: 4 April 2020

(C) The Author(s) 2020

\begin{abstract}
Aims/hypothesis Antigen-specific therapy aims to modify inflammatory T cell responses in type 1 diabetes and restore immune tolerance. One strategy employs GAD65 conjugated to aluminium hydroxide (GAD-alum) to take advantage of the T helper (Th)2-biasing adjuvant properties of alum and thereby regulate pathological Th1 autoimmunity. We explored the cellular and molecular mechanism of GAD-alum action in the setting of a previously reported randomised placebo-controlled clinical trial conducted by Type 1 Diabetes TrialNet.

Methods In the clinical trial conducted by Type 1 Diabetes TrialNet, participants were immunised with $20 \mu \mathrm{g}$ GAD-alum (twice or three times) or alum alone and peripheral blood mononuclear cell samples were banked at baseline and post treatment. In the present study, GAD-specific T cell responses were measured in these samples and GAD-specific T cell lines and clones were generated, which were then further characterised.

Results At day 91 post immunisation, we detected GAD-specific IL-13 ${ }^{+}$CD 4 T cell responses significantly more frequently in participants immunised with GAD-alum ( $71 \%$ and $94 \%$ treated twice or three times, respectively) compared with those immunised with alum alone $(38 \% ; p=0.003$ and $p=0.0002$, respectively) accompanied by high secreted levels of IL-13, IL-4 and IL-5, confirming a GAD-specific, GAD-alum-induced Th2 response. Of note, GAD-specific, IL-13 ${ }^{+}$CD4 $\mathrm{T}$ cells observed after immunisation co-secreted IFN- $\gamma$, displaying a bifunctional Th1/Th2 phenotype. Single-cell transcriptome analysis identified IL13 and IFNG expression in concert with the canonical Th2 and Th1 transcription factor genes GATA3 and TBX21, respectively. T cell receptor $\beta$-chain (TCRB) CDR3 regions of GAD-specific bifunctional T cells were identified in circulating naive and central memory CD4 T cell pools of non-immunised participants with newonset type 1 diabetes and healthy individuals, suggesting the potential for bifunctional responses to be generated de novo by GAD-alum immunisation or via expansion from an existing public repertoire.

Conclusions/interpretation GAD-alum immunisation activates and propagates GAD-specific CD4 T cells with a distinctive bifunctional phenotype, the functional analysis of which might be important in understanding therapeutic responses.
\end{abstract}

Keywords Autoreactive $\cdot$ Epitopes $\cdot$ GAD $\cdot$ GAD-alum · Glutamic acid decarboxylase $\cdot$ IL-13 $\cdot$ Immunotherapy $\cdot$ T cell receptor · T cells $\cdot$ TCR $\cdot$ Th2 $\cdot$ TrialNet

Electronic supplementary material The online version of this article (https://doi.org/10.1007/s00125-020-05130-7) contains peer-reviewed but unedited supplementary material, which is available to authorised users.

Mark Peakman

mark.peakman@kcl.ac.uk

1 Peter Gorer Department of Immunobiology, King's College London Faculty of Life Sciences and Medicine, 2nd Floor, Borough Wing, Guy's Hospital, London SE1 9RT, UK

2 Division of Endocrinology, Department of Pediatrics, The Hospital for Sick Children, University of Toronto, Toronto, ON, Canada
3 Homer Stryker MD School of Medicine, Western Michigan University, Kalamazoo, MI, USA

4 Diabetes and Metabolism Research Institute, City of Hope, Duarte, CA, USA

5 King's Health Partners Institute of Diabetes, Endocrinology and Obesity, King's College Hospital NHS Foundation Trust, London, UK 


\section{Research in context}

\section{What is already known about this subject?}

- Antigen-specific immunotherapy (ASI) is a commonly used, well-tolerated strategy to prevent type 1 diabetes

- ASI using GAD conjugated to alum (GAD-alum) did not result in clinical benefit

\section{What is the key question?}

- Why did immunotherapy with GAD fail in terms of clinical efficacy, despite eliciting powerful immunological responses?

\section{What are the new findings?}

- The T cell response induced upon immunisation with GAD-alum is not a polarised Thelper cell (Th) 2 response as expected but a bifunctional Th1/Th2 response, as these cells harbour features of proinflammatory Th1 cells

- These bifunctional Th1/Th2 cells are attenuated in any form of counter-regulatory function and therefore do not sufficiently divert the pathogenic autoimmunity responsible for type 1 diabetes progression and may explain the lack of clinical efficacy of GAD-alum

\section{How might this impact on clinical practice in the foreseeable future?}

- This study highlights the importance of investigating the mechanism of action of ASI as this may explain the lack of clinical efficacy

$\begin{array}{ll}\text { Abbreviations } \\ \text { Alum } & \text { Aluminium hydroxide } \\ \text { ASI } & \text { Antigen-specific immunotherapy } \\ \text { GADA } & \text { GAD autoantibodies } \\ \text { HA } & \text { Haemagglutinin } \\ \text { PBMC } & \text { Peripheral blood mononuclear cell } \\ \text { PE } & \text { Phycoerythrin } \\ \text { PHA } & \text { Phytohaemagglutinin } \\ \text { PMA } & \text { Phorbol 12-myristate 13-acetate } \\ \text { Th } & \text { T helper cell } \\ \text { TCGF } & \text { T Cell growth factor } \\ \text { TCR } & \text { T cell receptor } \\ \text { TCRB } & \text { T cell receptor } \beta \text {-chain }\end{array}$

\section{Introduction}

Type 1 diabetes develops in multiple stages preceded by immune cell activation and beta cell destruction. Stage 1 is characterised by the presence of autoantibodies targeting islet beta cell autoantigens, dysglycaemia is evident in stage 2 and stage 3 is characterised by symptomatic diabetes [1]. Therapeutic intervention to maintain beta cell function can be applied depending on whether the aim is prevention (prior to stage 1) or intervention (stages 1-3). Intervention studies include both antigen-specific and non-antigen-specific therapies.

Antigen-specific immunotherapy (ASI) has emerged as an attractive strategy for the prevention of type 1 diabetes development and loss of beta cell function. Based on successes in preclinical models, several early-stage clinical trials have been conducted using whole antigens presented orally, or antigens or peptides given by parenteral injection [2]. Although the results of these trials have been largely negative, most have been small and conducted at early stages of drug development on small numbers of individuals and have established that, according to current experience, ASI is very well tolerated and has a good safety profile [3].

One of the most advanced strategies in terms of drug development is the use of the autoantigen GAD65 conjugated to alum (GAD-alum) administered at low dose $(20 \mu \mathrm{g})$ via the subcutaneous route. While an early Phase I/II study showed encouraging prevention of C-peptide decline in individuals with recent-onset type 1 diabetes, a Phase II study conducted by Type 1 Diabetes TrialNet did not find any indication of efficacy [4] and neither did a small study in high-risk, multiple-antibody-positive individuals [5]. Finally, a metaanalysis of published results in people with new-onset type 1 diabetes does not entirely rule out a therapeutic benefit from the GAD-alum injections [6], and therefore the question of the setting in which this approach might be used remains unresolved.

While clinical efficacy has not been demonstrated, there is little doubt that immunisation with GAD-alum has powerful immunological effects. A series of mechanistic studies accompanied the clinical trials conducted to date and demonstrated that GAD-alum injection is a potent inducer of high-titre antiGAD65 autoantibodies and also results in changes in CD4 T 
lymphocyte activation, tissue homing receptor expression and cytokine release in response to stimulation with GAD65 in vitro $[7,8]$. The overarching interpretation of these results is that the balance of CD4 T lymphocyte responses to GAD65 is shifted by therapy away from $\mathrm{T}$ helper (Th) 1 and towards a Th2 response. Indeed, this was the original rationale for the approach of using alum as an adjuvant for GAD65, since it is known to bias CD4 $\mathrm{T}$ lymphocyte responses against coadministered antigens towards Th2 [9-11]. GAD-alum immunotherapy thus aimed to limit Th1-driven disease progression through the induction of autoantigen-specific Th2 responses, although evidence of this lacking.

These mechanistic outcomes present a conundrum, since while the immunological endpoints have largely been achieved in GAD-alum immunotherapy studies, this has not been accompanied by therapeutic benefit. In the present study we address the key question of whether the Th2 mechanistic rationale for GAD-alum immunotherapy is supported.

\section{Methods}

Participants We studied 46 participants with type 1 diabetes recruited for the previously reported Type 1 Diabetes TrialNet TN08 study ('Effects of Recombinant Human Glutamic Acid Decarboxylase (rhGAD65) Formulated in Alum (GAD-alum) on the Progression of Type 1 Diabetes in New Onset Subjects', ClinicalTrials.gov no. NCT00529399) from 15 sites in the USA and Canada within 100 days of diagnosis; participants were diagnosed with type 1 diabetes according to American Diabetes Association criteria (Electronic supplementary material [ESM] Table 1) ([4]. The Type 1 Diabetes TrialNet TN08 study was a three-arm, multicentre, randomised, double-masked, placebo-controlled clinical trial designed to test the hypothesis that multiple injections of GAD-alum preserve endogenous insulin production in type 1 diabetes. The primary outcome was to establish whether C-peptide in the GAD-alum-treated group differed significantly from the placebo-treated group at 1 year.

At entry, all participants had GAD65 antibodies (GADA) and stimulated C-peptide concentrations of $\geq 0.2 \mathrm{nmol} / 1$ during a mixed-meal tolerance test (MMTT). Participants received three subcutaneous injections of GAD-alum or alum (Diamyd, Diamyd Medical, Stockholm, Sweden) at baseline (day 0), day 28 and day 56, as previously described [4]; specifically, 16 out of 46 participants received GAD-alum injections three times, 17 received GAD-alum twice and then one injection of alum and 13 received alum three times. Samples obtained at baseline and 3 months post treatment (i.e. 3 months after the baseline treatment) were banked and used in the present study, which aimed to address the lack of therapeutic benefit in this GAD-alum trial. Samples from three of the 46 participants (one treated with GAD-alum twice and two treated three times) together with a further individual (treated with GAD-alum three times; additional samples shipped by Trialnet, as we required more cells for the subsequent experiments) were used in subsequent $\mathrm{T}$ cell line generation and epitope-mapping studies. In addition to these participants, we studied GAD-specific IFN- $\gamma$ responses in 71 non-trial individuals with recent-onset type 1 diabetes (47 men, mean age: 28.7 years, range: $18-46$ years, duration of disease: 0.25 14.5 months) (ESM Table 2). These individuals were recruited via the 'T cell studies in Type 1 diabetes' study at Guy's and St Thomas' Hospital and Bristol Royal Infirmary. Informed consent was obtained from all participants and this study was approved by the UK National Research Ethics Committee.

Detection of GAD-specific CD4 T cells Cryopreserved peripheral blood mononuclear cells (PBMCs) from GAD-alumand alum-immunised participants were thawed and stimulated with $10 \mu \mathrm{g} / \mathrm{ml}$ T cell GAD (recombinant human GAD expressed in baculovirus and formulated specifically for use in T cell assays) and GAD diluent (Diamyd Medical). Cultures were then examined for IL-13 responses by indirect ELISPOT (U-Cytech, Utrecht, Netherlands), as previously described [12]; in brief, IL-13 production by CD4 $\mathrm{T}$ cells was assessed by operators blinded to the immunisation status of the donor (GAD-alum or alum). Data are expressed as the mean number of spots per triplicate compared with the mean spot number in the presence of diluent alone (stimulation index [SI]). A receiver operating characteristic (ROC) plot was used to define optimal sensitivity and specificity and a cut-off of $\geq 2.5$ defined as a positive response (sensitivity $73 \%$, specificity $77 \%$ ). Supernatants from PBMCs cultured with T cell GAD for $24 \mathrm{~h}$ during these ELISpot assays were assessed for the presence of IFN- $\gamma$, IL-13, IL-4, IL-5, IL-10, IL-17 and IL-6 by Luminex (Millipore UK, Watford, UK), with standard curves generated with a dynamic range between 3 and $10,000 \mathrm{pg} / \mathrm{ml}$. Data were analysed using the Flexmap 3D software (R\&D systems, Abingdon, UK).

In some assays, cytokine responses were measured using combined IL-13/IFN- $\gamma$ indirect FluoroSpot assays (UCytech, Utrecht, the Netherlands) to examine cytokine cosecretion. Briefly, cells stimulated with GAD65, GAD peptide or the positive control (Pediacel, Sanofi, Guildford, Surrey) were transferred to wells of a pre-treated FluoroSpot plate coated with high affinity anti-IL-13 and anti-IFN- $\gamma$ antibodies [12]. After $24 \mathrm{~h}$ the cells were removed by washing, and antibody-bound cytokine identified using a mixture of anti-IL-13 and anti-IFN- $\gamma$ detection antibodies followed by two fluorescent conjugates containing Alexa 488-labelled anti-FITC antibodies and R-phycoerythrin (R-PE)-labelledstreptavidin made up according to manufacturer's instructions. Green fluorescent spots represent IFN- $\gamma$ producing 
cells, red spots, IL-13 or IL-4 and yellow spots signify cells that release both IFN- $\gamma$ and IL-13/IL-4.

T cell lines and clones In order to generate GAD-specific T cell lines, cryopreserved PBMCs from participants (3 months post immunisation with GAD-alum) were thawed and stimulated with $10 \mu \mathrm{g} / \mathrm{ml} \mathrm{T}$ cell GAD for $48 \mathrm{~h}$; IL-13-secreting cells were captured using an IL-13 secretion/detection kit for $1 \mathrm{~h}$ at $37^{\circ} \mathrm{C}$ (Miltenyi Biotec, Bisley, Surrey, UK). Cells were subsequently labelled with mouse anti-human IL-13-biotin followed by anti-biotin PE in a cocktail containing monoclonal mouse anti-human CD3 APC-H7 (BD Biosciences Cat. No. 560275, RRID:AB 1645476), CD14 V500 (Cat. No. 541391), CD19 V500 (Cat. No. 561121), CD8 FITC (BD Biosciences Cat. No. 555634, RRID:AB 395996) (Becton Dickinson, Oxford, UK) and CD4 allophycocyanin (APC) (BioLegend Cat. No. 317415, RRID:AB_571944) (BioLegend, London, UK) all made up according to manufacturer's instructions for $10 \mathrm{~min}$ on ice. Following a washing step, the cells were magnetically labelled with anti-PE microbeads, and a viability marker, 7-amino-actinomycin D (BioLegend, London, UK), was added. The cells were washed, and magnetic separation was performed using MS MACS columns (Miltenyi Biotec, Bisley, Surry, UK) to enrich for IL- $13^{+}$cells. CD4 $4^{+} \mathrm{IL}-13^{+} \mathrm{T}$ cells were then bulk sorted (typically 100-3000 cells were sorted per condition) using a FACSARIA II SORP cell sorter (Becton Dickinson) into polypropylene tubes containing $10^{5}$ irradiated mixed allogeneic PBMCs with $4 \mu \mathrm{g} / \mathrm{ml}$ phytohaemagglutinin (PHA) (Alere, Zug, Switzerland) in X-vivo-15 (Lonza, Slough, UK) containing 5\% (wt/vol.) human AB serum (Sigma Aldrich, Gillingham, Dorset, UK). The next day, fresh medium supplemented with $\mathrm{T}$ cell growth factor (TCGF; final concentration 5\% (wt/vol.); Cellkine, ZeptoMetrix, Buffalo, NY, USA) was added and cells were expanded in culture medium containing decreasing concentrations of TCGF for 2 weeks before testing for specificity by IL-13 ELISpot. In brief, GAD specificity was examined by direct IL-13 ELISpot (U-Cytech, Utrecht, the Netherlands) using $10^{4} \mathrm{~T}$ cells in the presence of $2 \times 10^{5}$ HLA-matched PBMCs for antigen presentation and $10 \mu \mathrm{g} / \mathrm{ml}$ T cell GAD. Epitope specificity of lines was examined using the same approach and individual peptides from a library of 115 overlapping peptides of GAD65 (20-mers overlapping by five amino acids; $10 \mu \mathrm{g} / \mathrm{ml}$; Thermo Hybaid, Ulm, Germany;) were used as stimulation. Peptides eliciting a positive IL-13 response (spot number $\geq$ the inter-assay mean of the diluent +3 SD) were re-screened twice to confirm the result. In addition, as controls, Th1 and Th2 cell lines were generated using specific differentiating protocols and human cellXvivo Th1 and Th2 cell differentiation kits as instructed by the manufacturer (R \& D systems, Abingdon, UK).

For the isolation of GAD-specific T cell clones, GADreactive $\mathrm{T}$ cell lines were stimulated with $10 \mu \mathrm{g} / \mathrm{ml} \mathrm{T}$ cell
GAD, labelled as above and $\mathrm{CD} 4^{+} \mathrm{IL}-13^{+}$cells were singlecell sorted into 96 well U-bottom plates containing $10^{5}$ irradiated mixed allogeneic PBMCs and PHA $(4 \mu \mathrm{g} / \mathrm{ml})$ in $200 \mu \mathrm{l}$ complete X-VIVO media containing 5\% (wt/vol.) human AB serum. The following day, the clones were supplemented with $10 \%$ (wt/vol.) TCGF and expanded in culture media containing decreasing concentrations of TCGF for 2 weeks. Clones and lines were expanded and maintained by alternate rounds of restimulation using PHA or T cell GAD and irradiated mixed allogeneic PBMCs.

In some experiments, intracellular cytokine staining was performed on GAD-specific clones and for comparison a haemagglutinin (HA)-peptide-specific flu clone following stimulation with phorbol 12-myristate 13-acetate (PMA)/ ionomycin (Sigma Aldrich, Gillingham, UK) at $50 \mathrm{ng} / \mathrm{ml}$ and $1 \mu \mathrm{g} / \mathrm{ml}$, respectively, or T cell GAD $(10 \mu \mathrm{g} / \mathrm{ml}$; Diamyd, Sweden) or GAD peptide (residues 555-567) or HA peptide (residues 306-318), accordingly (Thermo Hybaid, Ulm, Germany) at $10 \mu \mathrm{g} / \mathrm{ml}$, followed by monensin and brefeldin A (Becton Dickinson) for $3 \mathrm{~h}$ at $37^{\circ} \mathrm{C}$. The cells were then harvested and washed in PBS (Life Technologies, Paisley, UK) and stained with a live/dead marker (Aqua dead cell stain; ThermoFisher Scientific, Hemel Hempstead, UK) and following another wash in PBS, membrane staining was performed using mouse anti-human CD14 Pacific Blue (PB) (Thermo Fisher Scientific Cat. No. MHCD1428, RRID:AB_10373537), CD19 PB (Thermo Fisher Scientific Cat. No. MHCD1928, RRID:AB_10373689) (ThermoFisher Scientific), CD3 APC-H7 (Cat. No. 560275, RRID:AB_1645476, Becton Dickinson), CD4 PE-Cy5.5 (Cat. No. 560650, ThermoFisher Scientific) and CD8 PE-Cy7 (Cat. No. 344712 BioLegend) all made up according to manufacturer's instructions for $20 \mathrm{~min}$ at $4^{\circ} \mathrm{C}$ according to manufacturers' instructions. Cells were washed and fixed in CytoFix (Becton Dickinson) for $20 \mathrm{~min}$ at room temperature in the dark and subsequently permeabilised with permeabilisation solution (BioLegend) before intracellular staining using anti-human IL-13 APC (Cat. No. 501908), IL-4 FITC (Cat. No. 500807) (BioLegend) and anti-IFN- $\gamma$ PE (Cat. No. 502508) (Becton Dickinson) at room temperature for $20 \mathrm{~min}$. The cells were washed and resuspended for acquisition using a FACSCanto II flow cytometer (Becton Dickinson) and analysed using FlowJo v.10 (FlowJo, Ashland, OR, USA).

T cell receptor single-cell sequencing and cytokine analysis of GAD-specific $T$ cell lines and clones $T$ cell lines and clones were stimulated with $10 \mu \mathrm{g} / \mathrm{ml} \mathrm{T}$ cell GAD or peptide or DMSO as a control for $48 \mathrm{~h}$ and IL-13 or IL-13/IFN- $\gamma$ secreting cells captured using secretion/detection kits (Miltenyi Biotec, Bisley, UK) followed by sorting. Single-cell T cell receptor (TCR) sequencing and gene expression were performed as previously described [13, 14]. Sequencing 
results were annotated using the IMGT database [15], the CDR3A and CDR3B sequences extracted and compared across individual cells using KNIME 2.11.2 [16] and further analysed with R (version 3.4) (www.R-project.org/) and R package tcR [17] to determine identity with sequences in a repository of TCR sequences from circulating naive and central memory $\mathrm{CD} 4^{+} \mathrm{T}$ cells subsets $\left(>2 \times 10^{8}\right.$ total sequences from 14 type 1 diabetes subjects and 17 healthy control individuals) [18].

For the analysis of gene expression, $10 \mu \mathrm{l}$ of the first PCR product generated above were digested with exonuclease (ThermoFisher Scientific) and $1 \mu \mathrm{l}$ was added to diethyl pyrocarbonate (DEPC)-treated water, $2 \times$ SYBR Premix Ex Taq (Takara, Saint-Germain-en-Laye, France) and forward and reverse primer mixes for each of the six genes (IL13, GATA3, IL4, IFNG, TBX21 and SRP14 as a control gene). Primer sequences listed in [13] and in ESM Table 3 were added and samples analysed in the ABI PRISM 7900HT sequence detection system qPCR Real-Time PCR machine $\left(50^{\circ} \mathrm{C}\right.$ for $2 \mathrm{~min} ; 95^{\circ} \mathrm{C}$ for $10 \mathrm{~min} ;\left[95^{\circ} \mathrm{C}\right.$ for $15 \mathrm{~s} ; 60^{\circ} \mathrm{C}$ for $1 \mathrm{~min}] \times 40$ cycles; $95^{\circ} \mathrm{C}$ for $15 \mathrm{~s} ; 60^{\circ} \mathrm{C}$ for $15 \mathrm{~s} ; 95^{\circ} \mathrm{C}$ for $15 \mathrm{~s}$ [ramp rate of 2\%]). When the melting temperature of the amplified product was $\pm 1^{\circ} \mathrm{C}$ of that of the positive control (cDNA from $\mathrm{CD}^{+}$cells), it was considered that the template of interest was present in the sample. Subsequently, $\mathrm{C}_{\mathrm{t}}$ values were transformed into expression values $(\mathrm{E})$ by subtracting them from $40\left(E=40-C_{t}\right)$, so higher values mean higher expression.

Statistical analysis The frequency of responses and responses examining fold changes in alum- and GAD-alum-treated participants were compared using Mann-Whitney $U$ tests. ELISpot and cytokine responses at baseline vs post immunisation were analysed by Wilcoxon matched-pairs signed rank tests using GraphPad Prism software (version 8.3.1) Windows, GraphPad Software, San Diego, California USA, (www.graphpad.com). A $p$ value of $<0.05$ was considered significant. Association between variables was assessed with Spearman's rank correlation.

\section{Results}

GAD-specific Th2 responses are induced in GAD-alum-treated patients Individuals receiving GAD-alum or alum were examined for IL-13 production by ELISpot using PBMC samples obtained at baseline and day 91 by operators blinded to the treatment group. In baseline samples from all the participants, GAD-specific IL-13 responses are present at a low frequency in new-onset type 1 diabetes, with nine out of 46 (20\%) participants showing a response. GAD-alum immunotherapy resulted in a substantial increase in GAD-specific IL-13 responses at day 91 compared with baseline in participants receiving immunisations twice $(p=0.002)$ and three times $(p=0.0001)$ (Fig. 1a,b). In contrast, no change in GAD-specific IL-13 responses were seen in the participants who received placebo (alum alone). In participants treated with GAD-alum twice or three times, the fold change in IL-13 response from baseline was significantly greater compared with that in the placebo group ( $p=0.003$ and 0.0002 , respectively) (Fig. 1c). A total of 12 out of 17 (71\%; two GAD-alum injections) and 15 out of 16 (94\%; three GAD-alum injections) had a more than twofold increase in IL-13 response to GAD between baseline and day 91, compared with five out of 13 (38\%) participants treated with alum alone. Furthermore, the relationship between $\mathrm{Th} 2$ responsiveness and change in GADA titres was significant after GAD-alum treatment but was not significant after alum treatment (Fig. 1d,e).

There was no correlation between IL-13 responses and Cpeptide AUC (data not shown). We also examined whether GAD-specific IL-13 responses were influenced by HLA haplotypes, specifically HLA-DR3 and HLA-DR4. Six of the GADalum-treated participants possessed the HLA-DR3/DR3 haplotype and four of these showed a GAD-specific IL-13 response (67\%); 16 participants were positive for HLA-DR4 and of these $12(75 \%)$, had a GAD-specific IL-13 response (DR3 vs DR4, $p=1)$; nine were HLA-DR3/DR4 positive and eight of these ( $89 \%$ ) had a GAD-specific IL-13 response. In summary, there was no preferential association between HLA-DR haplotype and GAD-specific IL-13 responses.

Spontaneous cytokine secretion in response to $24 \mathrm{~h}$ of GAD65 stimulation in vitro was not observed in the baseline samples from all participants. However, at day 91 post immunisation in participants receiving GAD-alum twice there was a significant increase in secreted IL-5 $(p<0.05)$ and immunisation with GAD-alum three times induced a significant increase in secreted IL-13 $(p<0.01)$, IL-4 $(p<0.05)$, IL-5 $(p<0.01)$ and IL-17 $(p<0.05)$ (ESM Fig. 1$)$. There was no change in the placebo (alum alone) group. There was also no increase in the immune-regulatory cytokine IL-10 post immunisation in GAD-alum-treated participants.

Overall, our findings indicate that GAD-alum immunotherapy is associated with a robust, adaptive immune response involving an expansion of the number of circulating GADspecific $\mathrm{T}$ cells secreting the canonical Th2 cytokines IL-4, IL-5 and IL-13, directly linked to changes in the GADspecific humoral response.

T cell responses generated after GAD-alum immunisation target multiple GAD65 epitopes To characterise these responses further and to facilitate studies at single-cell resolution, we examined the epitope specificity of type 2 responses to GAD. T cell lines were generated from four GAD-alumimmunised participants; two lines were generated from participants homozygous for the HLA-DRBI*0301/DQA1*0501/ $D Q B 1 * 0201$ genotype, one from an individual homozygous 
a
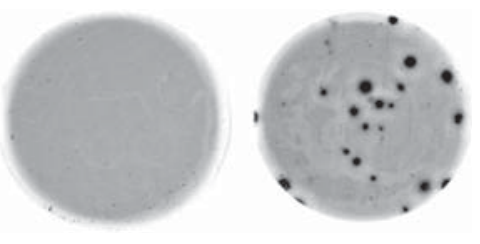

b

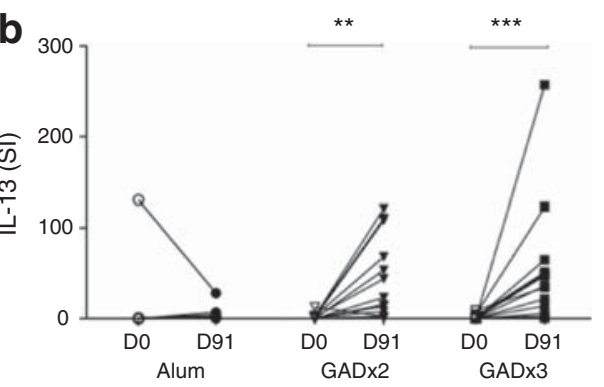

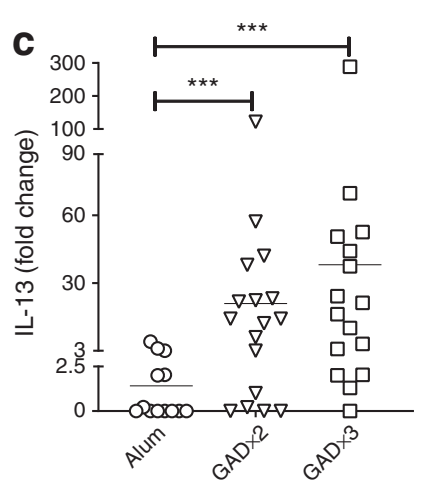

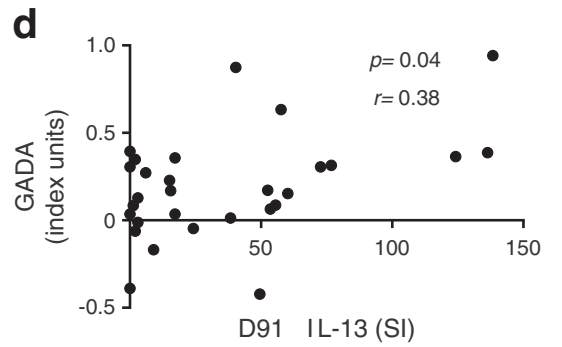

Fig. 1 Increase in GAD-specific IL-13 responses in GAD-alum-treated participants. (a) Representative ELISpot images from a GAD-alum-treated patient at baseline and at day 91 post treatment. Spots represent IL-13 production by single CD4 T cells. IL-13 responses are unusual in type 1 diabetes but are induced after GAD-alum immunotherapy, as demonstrated in (b) showing increased frequency of GAD-specific IL-13 responses post vaccination with $G A D$-alum (twice $[G A D \times 2]$ or three times [GAD $\times 3]$, but not with alum alone (IL-13 stimulation index [SI] at day 0 [D0; baseline, first treatment] and day 91 [D91]). SI represents the number of spots after stimulation in vitro with recombinant human GAD (rhGAD65) divided by the number of spots after control stimulation. Differences between D0 and D91 for each of treatment group are

for $H L A-D R B 1 * 0404 / D Q A 1 * 0301 / D Q B 1 * 0302$ and a further line from a heterozygous individual (HLA-DRB1*0301/ $D Q A 1 * 0501 / D Q B 1 * 0201 ; D R B 1 * 0401 / D Q A 1 * 0301 /$

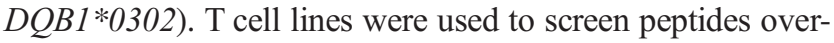
lapping the entire GAD65 sequence by IL-13 ELISpot assay. For each line, multiple epitopes were identified throughout the GAD65 sequence (Figs. 2 and 3).

For the $H L A-D R B 1 * 0301 / D Q A 1 * 0501 / D Q B 1 * 0201$ participant lines, 15 peptides were identified that elicited an IL-13 response, nine of which were nested around adjacent overlapping sequences (GAD226-245, GAD231-250, GAD281-300, GAD286-305, GAD371-390, GAD376-395, GAD556-575, GAD561-580, GAD566-585) (Fig. 2) and six represented single sequences (GAD81-100, GAD161-180, GAD420-445, GAD431-450, GAD511-530 and GAD531-550).

Five peptides were recognised by the HLA-DRBI*0404/ $D Q A 1 * 0301 / D Q B 1 * 0302$ participant line encompassing peptides GAD161-180, GAD211-230, GAD226-245, GAD241-260 and GAD381-400 (Fig. 2). Two of these peptides (GAD161-180 and GAD226-245) appear promiscuous as they were also targeted by the $H L A-D R B 1 * 0301 /$ $D Q A 1 * 0501 / D Q B 1 * 0201$ participant line.
For the heterozygous HLA-DR3/DR4 participant line, IL13 responses were detected against five peptides, three of which were adjacent overlapping sequences (GAD371-390, GAD376-395 and GAD381-400) and single peptides GAD281-300 and GAD461-480.

To summarise these findings, induced Th2 responses to GAD65 target multiple regions across the molecule, some of which overlap in individuals with different HLA genotypes.

T cells generated after GAD-alum immunisation display a bifunctional Th1/Th2 phenotype The ELISpot and cytokine secretion analyses show that GAD-alum immunisation generates a GAD-specific Th2 response. We and others have previously reported that GAD-specific Th1 responses are a feature of the natural history of type 1 diabetes $[12,19,20]$. Since the proposed mechanism of action of GAD-alum is immune diversion of autoreactive Th1 to Th2 responses, we next examined the fate of anti-GAD Th1 responses present at baseline and their relationship to the development of treatmentinduced anti-GAD Th2 responses, using a FluoroSpot assay that simultaneously detects secretion of both IFN- $\gamma$ and IL-13 on a single-cell-specific basis. 


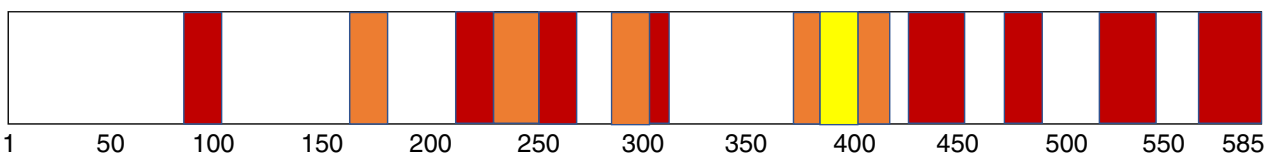

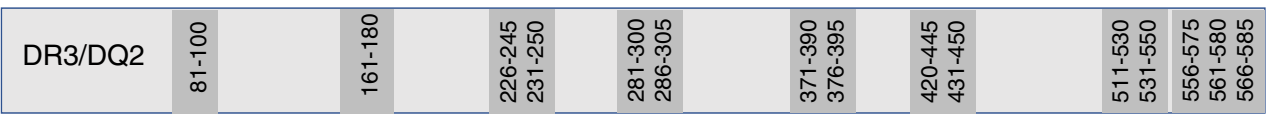

\begin{tabular}{|c|c|c|}
\hline DR4/DQ8 & $\begin{array}{l}\frac{0}{\div} \\
\frac{1}{6}\end{array}$ & 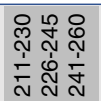 \\
\hline
\end{tabular}

DR3/4 DQ2/8

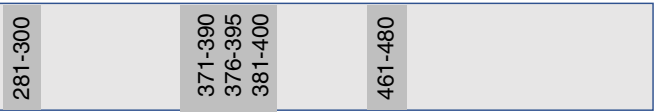

Fig. 2 Immunogenic regions of GAD65. Regions were mapped by screening overlapping GAD65 peptides with GAD-specific T cell lines generated using PBMCs from participants immunised with GAD-alum and tested for IL-13 response by ELISpot. Lines were generated from participants homozygous for HLA-DR3/DQ2 (DRB1*0301/

$D Q A 1 * 0501-D Q B 1 * 0201)$, homozygous for HLA-DR4/DQ8 $(D R B 1 * 0404 / D Q A 1 * 0301 D Q B 1 * 0302)$ and heterozygous for HLA$\mathrm{DR} 3 / 4 \mathrm{DQ} 2 / 8$. Epitope regions are shown in red when they are observed in only one line, orange when observed in two lines and yellow when multiple lines show reactivity

We confirmed previous findings, namely that a subset of individuals (31 out of $71 ; 44 \%$ ) tested at onset of type 1 diabetes is characterised by the presence of GAD-specific IFN- $\gamma$ secreting $\mathrm{T}$ cells, which are revealed after stimulation ex vivo with GAD65 (ESM Fig. 2). However, the most striking observation is that post GAD-alum immunisation, there is a population of IL-13-secreting T cells that also produce IFN- $\gamma$ which is absent from baseline samples (Fig. 4). IL-13 $3^{+}$ IFN- $\gamma^{+}$cells specific for GAD are significantly expanded in GAD-alum post-immunisation samples compared with baseline samples (e.g. for the peptides GAD226-245 and GAD556-575, $p=0.02$ and $p=0.03$, respectively) but not in placebo (alum) post-immunisation samples (Fig. 5; ESM Figs. $3,4)$. Dual-positive cells in response to the recall antigen

Pediacel did not change in number during the study. In contrast, the magnitude of single GAD-specific IFN- $\gamma$ or IL13 responses remained unchanged (GAD226-245) or declined significantly (GAD556-575) compared with baseline. In addition, GAD-specific IFN- $\gamma^{+} / \mathrm{IL}-13^{+}$-co-secreting CD4 T cells represent a stable phenotype, induced by GAD-alum, since they remained detectable in samples tested up to 12 months after immunisation ( $n=4$ individuals) (ESM Fig. 5). While these peptide-based studies provide strong confirmatory evidence of antigen specificity, in concert with the experiments using whole GAD65, it is possible that some of the responses reflect a degree of cross-reactivity, perhaps as a result of peptides binding to HLA molecules in unconventional registers.

\begin{tabular}{ccc}
\hline Region & Epitope & Sequence \\
\hline $81-100$ & $81-100$ & SCSKVDVNYAFLHATDLLP \\
$161-180$ & $161-180$ & MHCQTTLKYAIKTGHPRYFN \\
$211-260$ & $211-230$ & PVFVLLEYVTLKKMREIIGW \\
& $226-245$ & EIIGWPGGSGDGIFSPGGAI \\
& $231-250$ & PGGSGDGIFSPGGAISNMYA \\
& $241-260$ & PGGAISNMYAMMIARFKMFP \\
$281-305$ & $281-300$ & SHFSLKKGAAALGIGTDSVI \\
& $284-305$ & SLKKGAAALGIGTDSVILIKCD \\
$371-400$ & $371-390$ & LLMSRKHKWKLSGVERANSV \\
& $376-395$ & KHKWKLSGVERANSVTWNPH \\
$420-450$ & $381-400$ & LSGVERANSVTWNPHKMMGV \\
& $420-445$ & QMHASYLFQQDKHYDLSYDTGDALQ \\
$461-480$ & $431-450$ & KHYDLSYDTGDKALQCGRHV \\
$511-550$ & $461-480$ & AKGTTGFEAHVDKCLELLAEY \\
& $511-530$ & PSLRTLEDNEERMSRLSKVA \\
$556-585$ & $531-550$ & PVIKARMMEYGTTMVSYQPL \\
& $556-575$ & FFRMVISNPAATHQDIDFLI \\
& $561-580$ & ISNPAATHQDIDFLIEEIER \\
& $566-585$ & ATHQDIDFLIEEIERLGQDL \\
\hline
\end{tabular}

Fig. 3 GAD epitopes identified by screening overlapping peptides for IL-13 responses 


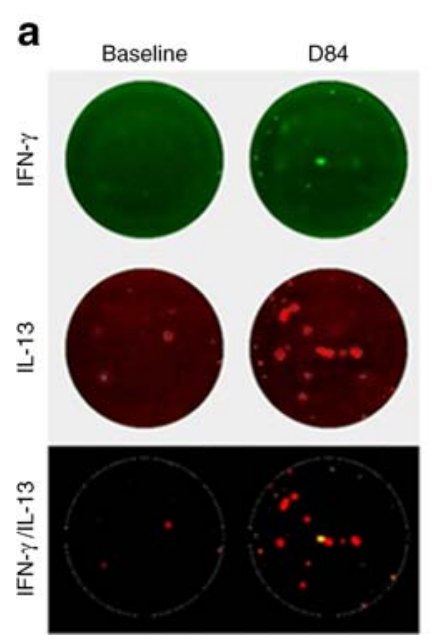

Fig. 4 Examples of GAD-specific bifunctional Th1/Th2 cells induced upon GAD-alum immunisation. Representative data from two GADalum-vaccinated participants showing responses to GAD65 peptides (a) 231-250 and (b) 556-575 at baseline and day 84 (D84) post immunisation analysed by dual IL-13/IFN- $\gamma$ indirect FluoroSpot. In the

\section{GAD-specific $T$ cell clones display bifunctionality To examine} the nature of GAD-specific IL-13-secreting T cells induced upon GAD-alum immunisation at single-cell resolution we generated 22 GAD-specific $T$ cell clones from a GADspecific $\mathrm{T}$ cell line from an immunised individual using a strategy that specifically enriches for $\mathrm{CD} 4^{+} \mathrm{IL}-13^{+}$cells. For 21 out of 22 clones we observed that IL-13 secretion was accompanied by IFN- $\gamma$ production following PMA/

Fig. 5 GAD-specific bifunctional cells are induced upon GADalum immunisation. IFN- $\gamma$ and IL-13 responses to GAD epitopes: GAD226-245 (a, e, i), GAD231-250 (b, f, j), GAD556-575 (c, g, k) and Pediacel $(\mathbf{d}, \mathbf{h}, \mathbf{l})$ were analysed by dual IL-13/IFN- $\gamma$ indirect FluoroSpot in nine participants immunised with GAD-alum at baseline $(\mathrm{B} / \mathrm{L})$ and at day 84 (D84). Bifunctional Th1/Th2 responses are shown in $(\mathbf{a}-\mathbf{d})$, single IL-13 in (e-f) and IFN- $\gamma$ responses in (i-I). Each symbol represents a different immunised participant. The comparison between responses at baseline and D84 post immunisation were analysed by Wilcoxon matchedpairs signed rank tests $(* p<0.05)$
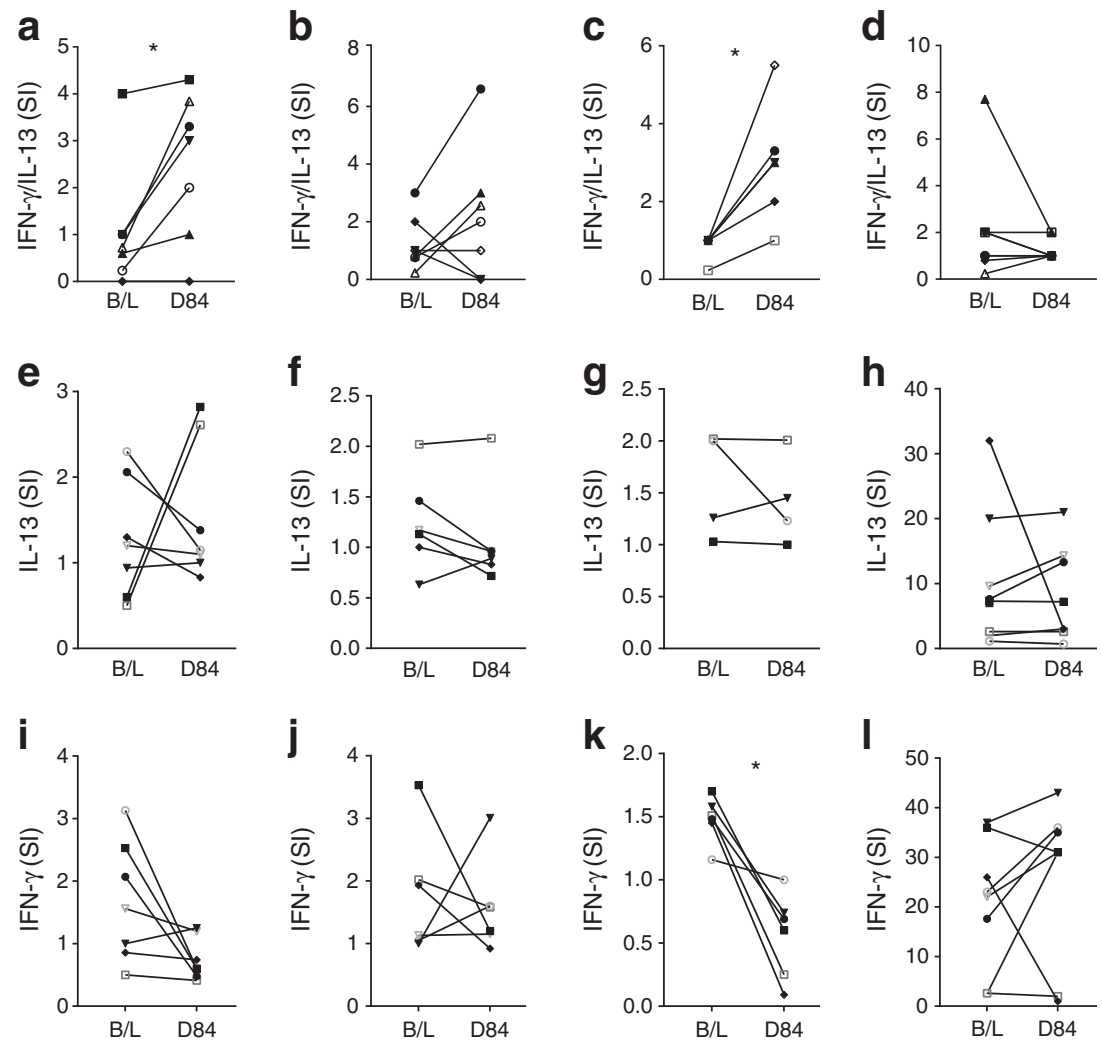

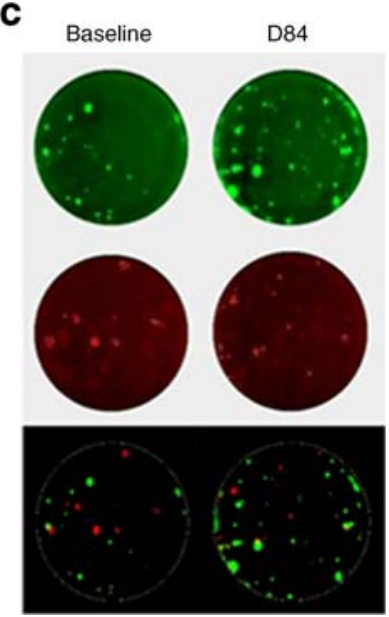

merged image panels, dual-cytokine-secreting cells appear yellow. (c) Baseline and day 84 responses to the recall antigen Pediacel for comparison. IFN- $\gamma$ responses are shown in green, IL-13 in red and bifunctional Th1/Th2 responses are yellow

ionomycin stimulation in vitro (Fig. 6a). A median of $15 \%$ (range 2-90\%) of cells in each of the clone populations coproduced both IL-13 and IFN- $\gamma$ in response to this polyclonal stimulus (not shown), and dual-cytokine production (IL-13 FN- $\gamma$ ) was also seen following stimulation with cognate tion, we examined expression of canonical markers of Th1 and Th2 differentiation at single-cell resolution at the 

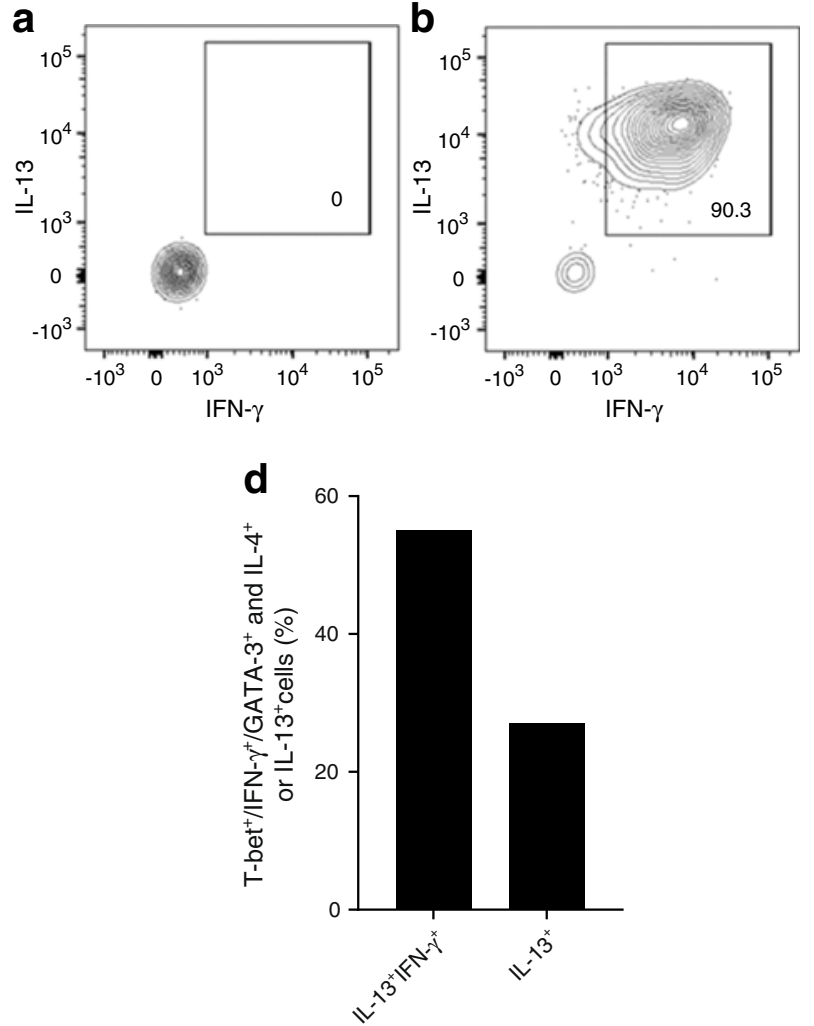

Fig. 6 GAD-specific CD4 T cells induced by GAD-alum immunisation are bifunctional. (a) Representative GAD-specific CD4 T cell clone stimulated with diluent DMSO or (b) PMA/ionomycin for $3 \mathrm{~h}$ and stained for intracellular expression of IL-13 and IFN- $\gamma$ shows the clone's bifunctional potential. (c) GAD peptide-specific clone exhibits a bifunctionality when stimulated with cognate GAD65 555-567 peptide in vitro and analysed by dual-cytokine FluoroSpot. The control condition is

transcriptional level (canonical Th1 transcription factor $T B X 21$ and cytokine IFNG; canonical Th2 transcription factor GATA3 and cytokines IL4, IL13). The majority of GAD65stimulated CD4 $\mathrm{T}$ cells from specific lines and clones exhibited a hybrid phenotype, expressing a combination of TBX21, IFNG and GATA3, as well as IL4 and/or IL13 (Fig. 6d); in contrast this hybrid phenotype was observed in $2 \%(1 / 41)$ and no $(0 / 42)$ pure Th1 and Th2 lines, respectively (not shown).

Th1/Th2 bifunctional T cells generated after GAD-alum immunisation originate from naive and memory compartments We hypothesised that GAD-specific bifunctional Th1/ Th2 cells could originate in two ways: following GAD-alum immunisation, naive $\mathrm{T}$ cells could be activated de novo under conditions that favour bifunctionality; or pre-existing GADspecific memory $\mathrm{T}$ cells could be expanded under conditions that favour deviation to bifunctionality. To begin to address this, we sequenced $\mathrm{T}$ cell receptor $\beta$-chain (TCRB) CDR3 regions of GAD-specific bifunctional $\mathrm{T}$ cells obtained from two of the T cell lines (one donor HLA-DRB1*0301/ $D Q A 1 * 0501 / D Q B 1 * 0201$ genotype, one HLA-DRB1*0404/

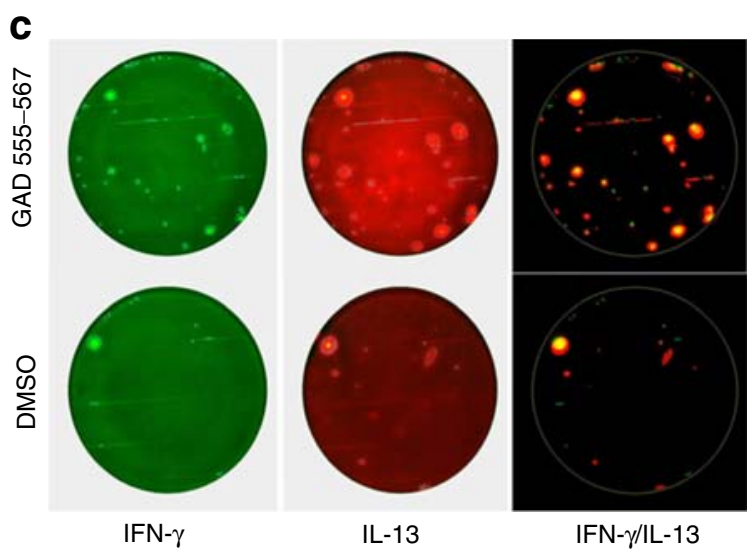

stimulation with DMSO (the peptide diluent). The Th1/Th2 phenotype $\left(\mathrm{IFN}-\gamma^{+} / \mathrm{IL}-13^{+}\right)$is shown in yellow. IFN- $\gamma$ responses are green and IL-13 are red (representative data from six clones). (d) Expression of cytokine and transcription factor mRNA by single sorted clone/line cells detected by PCR shows that a majority of GAD-alum induced bifunctional CD4 T cells co-express Th1 and Th2 differentiation markers TBX21 (encoding Tbet), IFNG and GATA3 as well as IL4 and/or IL13

$D Q A 1 * 0301 / D Q B 1 * 0302)$ and matched these against a repository of TCRB CDR3 sequences from circulating naive, and central memory $\mathrm{CD} 4^{+} \mathrm{T}$ cell subsets that we obtained previously from a separate cohort of non-GAD immunised, new-onset type 1 diabetes individuals and HLA-matched, non-diabetic healthy individuals [18]. A total of 61 discrete TCRB CDR3 region sequences were obtained from a total of 271 sorted GAD-specific bifunctional T cells. Of these 61, 20 had amino acid sequences that matched identically to sequences in our naive and central memory $\mathrm{CD} 4^{+} \mathrm{T}$ cell repository. Of these, 12 out of 20 were found in naive and central memory cells, six out of 20 were exclusively in naive cells and two out of 20 were exclusively in the central memory CD4 T cell populations in both participants with type 1 diabetes and healthy individuals (Fig. 7a,b). One TCRB CDR3 region (CASSLEVNTEAFF) was present in the naive pool in every individual screened, indicating that this is a highly public clonotype. The very public nature of the CASSLEVNTEAFF clonotype has been highlighted by others both at the amino acid [21,22] and nucleotide level (tgtgccagcagtttggaggtgaacactgaagctttcttt) (http:// 
Fig. 7 T cell receptors of Th1/ Th2 bifunctional cells. Th1/Th2 bifunctional cells induced upon GAD-alum immunisation have the potential to arise after priming from naive cells and/or expansion from GAD-specific central memory CD4 T cells. TCRB CDR3s from bifunctional cells from GAD-specific T cell lines generated post GAD-alum immunisation were sequenced; these were then compared against TCRB CDR3s bulk sequenced from naive and central memory $\mathrm{CD}^{+} \mathrm{T}$ cell subsets from participants with type 1 diabetes $(n=14)$ and healthy controls $(n=$ 17) (true naive, TN; central memory, CM) [18]. The CDR3s from bifunctional Th1/Th2 cells are identifiable in both (a) naive and (b) central memory cells. The probability of generation (pGEN) values are shown on the bottom of the panels: values ranging from $10^{-7}$ to $10^{-10}$ indicate a high chance of a TCR being generated and therefore likely to be public. (c) The prevalence of clonotypes amongst all participants
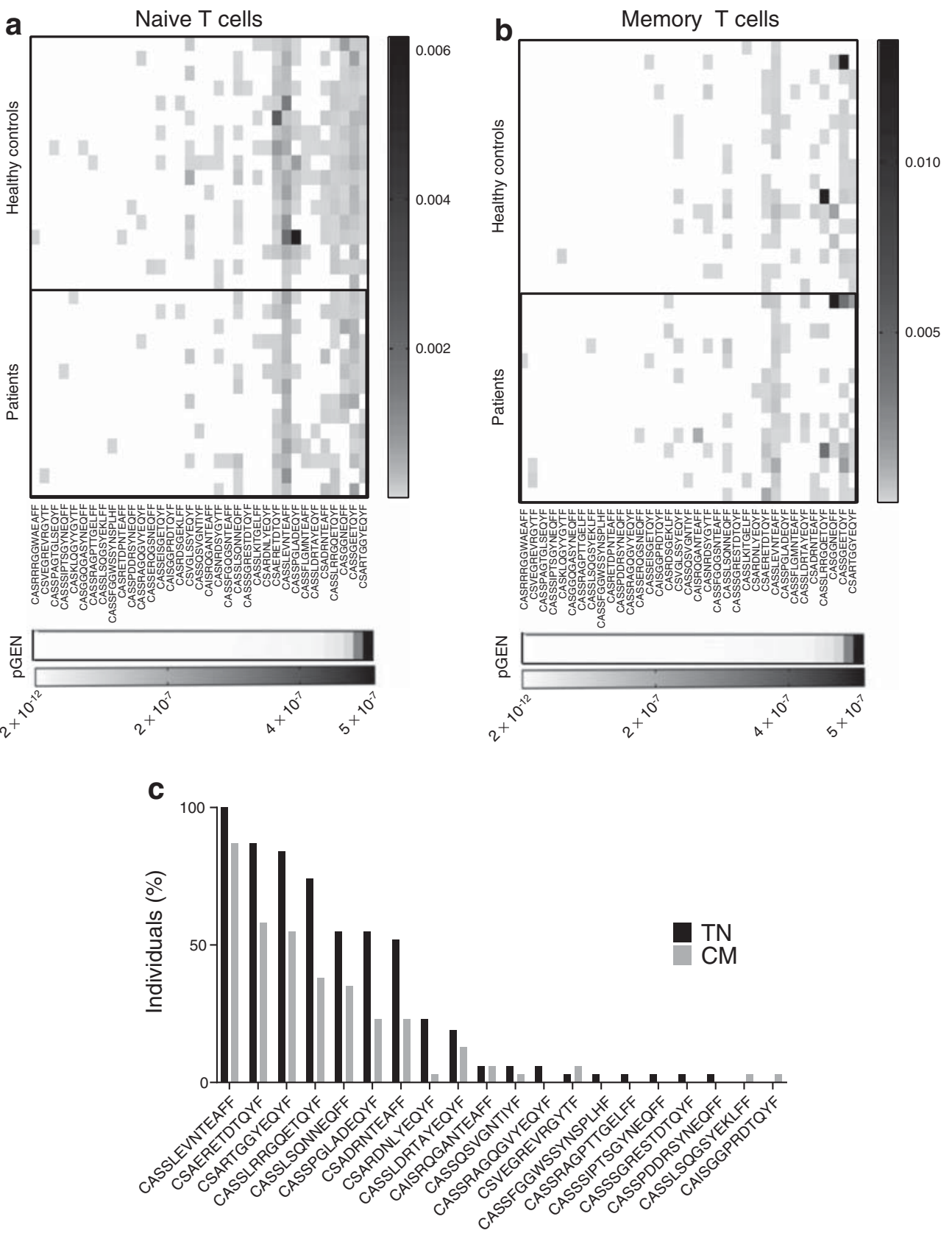

clonesearch.jdrfnpod.org/). The mean frequency of central memory clonotypes that shared TCRB CDR3s with GADspecific $\mathrm{T}$ cells is higher in individuals with type 1 diabetes than in healthy control individuals but not significant $(p=0.052)$ (data not shown).

With the caveat that we have only examined one chain of the TCR, these data suggest that T cells with the potential to recognise GAD epitopes in immunised patients are expanded from a highly public TCRB repertoire that may be present in both the naive and central memory cell pools in both individuals with type 1 diabetes and healthy individuals.

\section{Discussion}

GAD-alum immunotherapy has the stated aim of subverting $\mathrm{T}$ cell-mediated autoimmune destruction of islet beta cells by redirecting a predominant ('pathogenic') Th1 response towards a Th2 response. While immunological studies, including ours, indicate that Th2-like responses are indeed detectable after GAD-alum immunisation [7, 23], this change has not been unequivocally shown to translate into preservation of beta cell function or clinical benefit [4, 24]. In the present study we explored this disconnect between immunological and clinical efficacies. Through a detailed molecular 
and cellular examination of GAD-specific CD4 T cells induced by GAD-alum immunisation we demonstrate that a majority of the responder clones harbour features of both Th1 and Th2 cells and can thus be considered bifunctional. We speculate that this phenotype lacks immune-modulating properties and hence may not be capable of modifying autoimmunity in a clinically favourable way.

Bifunctional cells that share lineage and phenotypic features of two canonical CD4 T cell differentiation pathways have been described previously in autoimmune disease and in other settings. Th1/Th2 bifunctional cells were reported in humans in progressive multifocal leucoencephalopathy immune reconstitution inflammatory syndrome (PML-IRIS), a complication of monoclonal antibody therapy in multiple sclerosis. CD4 T cells isolated from brain tissue were found to secrete both IL- 4 and IFN- $\gamma$ and express GATA- 3 and T-bet (encoded by TBX21) [25]. Our report of GAD-specific Th1/ $\mathrm{Th} 2$ bifunctional CD4 $\mathrm{T}$ cells both upholds and extends these observations, since we not only confirm the presence of these cells in the circulation, but we also identify them as autoreactive, which to our knowledge has not been reported before. Other studies have suggested bifunctionality of cells co-expressing IFN- $\gamma$ and IL-17 in humans with inflammatory bowel disease and systemic lupus erythematosus [26, 27]. We established the presence of bifunctional IL-4/IL-13 ${ }^{+}$IFN- $\gamma^{+}$ cells induced upon GAD-alum immunisation at single-cell resolution for both protein production and gene expression using assays that measure responses directly ex vivo. Th1/ Th2 cells were present in approximately two-thirds of participants treated with GAD-alum. Moreover, we demonstrated that GAD-specific CD4 T cell clones and lines derived from treated participants after expansion in vitro co-produce both IL-13 and IFN- $\gamma$ and express the Th 2 cytokine IL- 4 as well as the canonical transcription factors (T-bet and GATA-3) for Th1 and Th2 cells, respectively. Participants treated with alum alone rarely presented an IL-13 response. Once induced by immunisation, bifunctional GAD-specific Th1/Th2 CD4 T cells are robustly stable for up to 12 months, a feature that has also been reported by others [28]. Interestingly, increased GAD-specific IFN- $\gamma$ and IL-4 responses by ELISpot have been reported previously in GAD-alum-treated participants [29], but in this case it was not established whether these were produced simultaneously by the same cell.

Bifunctional Th1/Th2 CD4 T cells may arise in vivo during infections and display functional properties that are quantitatively intermediate between Th1 and Th2 cells [30]. As such, they are considered to be attenuated in immunological potency, cause less immunopathology, and have a self-limiting role perhaps designed to limit excessive inflammation [30]. A similar self-limiting function has been assigned to skinresident Th17 cells that are poised to adopt a Th2 phenotype in cases of tissue injury or impaired regulation which licenses type 2 immunity and thereby promotes tissue repair [31].
Collectively, as our observations based on stable cytokine secretion indicate, bifunctional CD4 T cells represent a defined subpopulation of differentiated cells rather than an intermediate stage.

How a population of Th1/Th2 cells arises upon GAD-alum immunisation is open to speculation. As demonstrated in the present study and previously reported by others [29, 32], multiple cytokines are detected in the supernatants of GADstimulated cultures from GAD-alum-immunised participants, and it is possible that if these conditions are reproduced in tissues in vivo upon immunisation, this distinctive cytokine milieu is a potent imprinting mechanism, either redirecting pre-existing GAD-specific Th1 or, more rarely Th2 cells; or inducing bifunctionality de novo. Of note, we did not find any evidence that the bifunctional response was focused onto any particular immunogenic regions of the GAD65 molecule, but this may be worthy of further analysis and fine-mapping of responses at the clonal level.

Finally, we attempted to address the question as to whether expanded bifunctional Th1/Th2 cells derive from the antigeninexperienced or naive $\mathrm{CD} 4 \mathrm{~T}$ cell pool. By searching for TCRB CDR3 sequences from induced bifunctional CD4 T cell clone responses in a repository of TCR sequences from circulating naive and central memory $\mathrm{CD}^{+} \mathrm{T}$ cell subsets from individuals with new-onset type 1 diabetes and healthy individuals we were able to show that numerous GADspecific clonotype signatures are public, being present in multiple people; and not disease-specific, being present in both those with diabetes and the healthy control individuals. We observed that TCRB CDR3 clonotypes were present at a greater frequency in the central memory pool in individuals with type 1 diabetes, suggesting that they are preferentially expanded as part of the disease process.

Thus, CD4 T cells recognising GAD through a distinctive, bifunctional differentiation pathway are expanded numerically post immunisation on the background of a potentially facilitating, highly public and disease-agnostic TCRB repertoire. The relevance of this process to the equivocal clinical outcomes seen in proof-of-concept efficacy studies is unclear, but it is reasonable to hypothesise that bifunctional cells are attenuated in any form of counter-regulatory function and thus do not sufficiently divert the pathogenic autoimmunity responsible for type 1 diabetes progression.

Acknowledgements We acknowledge the support of the Type 1 Diabetes TrialNet Study Group, which identified study participants and provided samples and follow-up data for this study.

Data availability The data are available upon request from the authors.

Funding The Type 1 Diabetes TrialNet Study Group is a clinical trials network funded by the National Institutes of Health $(\mathrm{NIH})$ through the National Institute of Diabetes and Digestive and Kidney Diseases, the National Institute of Allergy and Infectious Diseases, and The Eunice Kennedy Shriver National Institute of Child Health and Human 
Development, through the cooperative agreements U01 DK061010, U01 DK061016, U01 DK061034, U01 DK061036, U01 DK061040, U01 DK061041, U01 DK061042, U01 DK061055, U01 DK061058, U01 DK084565, U01 DK085453, U01 DK085461, U01 DK085463, U01 DK085466, U01 DK085499, U01 DK085505, U01 DK085509 and JDRF. The contents of this article are solely the responsibility of the authors and do not necessarily represent the official views of the NIH or JDRF.

Authors' relationships and activities The authors declare that there are no relationships or activities that might bias, or be perceived to bias, their work.

Contribution statement SA designed and performed experiments, analysed data and wrote the manuscript. IGT, YK, IPA, EH, DM and $\mathrm{CH}$ performed experiments, analysed data and reviewed the manuscript. $\mathrm{CB}$ and AL analysed data and reviewed the manuscript. SA and MP designed research studies. SA, TT, BR and MP conceived ideas and reviewed the manuscript. SA and MP oversaw research. DKW designed the study, analysed data and edited the manuscript. All authors gave final approval of the version to be published. MP is the guarantor of this work and, as such, had full access to all the data in the study and takes responsibility for the integrity of the data and the accuracy of the data analysis.

Open Access This article is licensed under a Creative Commons Attribution 4.0 International License, which permits use, sharing, adaptation, distribution and reproduction in any medium or format, as long as you give appropriate credit to the original author(s) and the source, provide a link to the Creative Commons licence, and indicate if changes were made. The images or other third party material in this article are included in the article's Creative Commons licence, unless indicated otherwise in a credit line to the material. If material is not included in the article's Creative Commons licence and your intended use is not permitted by statutory regulation or exceeds the permitted use, you will need to obtain permission directly from the copyright holder. To view a copy of this licence, visit http://creativecommons.org/licenses/by/4.0/.

\section{References}

1. Couper JJ, Haller MJ, Greenbaum CJ et al (2018) ISPAD clinical practice consensus guidelines 2018: stages of type 1 diabetes in children and adolescents. Pediatr Diabetes 19(Suppl 27):20-27. https://doi.org/10.1111/pedi.12734

2. Smith EL, Peakman M (2018) Peptide immunotherapy for type 1 diabetes — clinical advances. Front Immunol 9:392. https://doi.org/ 10.3389/fimmu.2018.00392

3. Roep BO, Wheeler DCS, Peakman M (2019) Antigen-based immune modulation therapy for type 1 diabetes: the era of precision medicine. Lancet Diabetes Endocrinol 7(1):65-74. https://doi.org/ 10.1016/s2213-8587(18)30109-8

4. Wherrett DK, Bundy B, Becker DJ et al (2011) Antigen-based therapy with glutamic acid decarboxylase (GAD) vaccine in patients with recent-onset type 1 diabetes: a randomised doubleblind trial. Lancet 378(9788):319-327. https://doi.org/10.1016/ S0140-6736(11)60895-7

5. Elding Larsson H, Lundgren M, Jonsdottir B, Cuthbertson D, Krischer J, DiAPREV-IT Study Group (2018) Safety and efficacy of autoantigen-specific therapy with 2 doses of alum-formulated glutamate decarboxylase in children with multiple islet autoantibodies and risk for type 1 diabetes: a randomized clinical trial. Pediatr Diabetes 19(3):410-419. https://doi.org/10.1111/pedi. 12611
6. Beam CA, MacCallum C, Herold $\mathrm{KC}$ et al (2017) GAD vaccine reduces insulin loss in recently diagnosed type 1 diabetes: findings from a Bayesian meta-analysis. Diabetologia 60(1):43-49. https:// doi.org/10.1007/s00125-016-4122-1

7. Axelsson S, Cheramy M, Akerman L, Pihl M, Ludvigsson J, Casas $\mathrm{R}$ (2013) Cellular and humoral immune responses in type 1 diabetic patients participating in a phase III GAD-alum intervention trial. Diabetes Care 36(11):3418-3424. https://doi.org/10.2337/dc122251

8. Axelsson S, Hjorth M, Ludvigsson J, Casas R (2012) Decreased $\mathrm{GAD}_{65}$-specific Th1/Tc1 phenotype in children with type 1 diabetes treated with GAD-alum. Diabet Med 29(10):1272-1278. https:// doi.org/10.1111/j.1464-5491.2012.03710.x

9. Brewer JM, Conacher M, Hunter CA, Mohrs M, Brombacher F, Alexander J (1999) Aluminium hydroxide adjuvant initiates strong antigen-specific Th2 responses in the absence of IL-4- or IL-13mediated signaling. J Immunol 163(12):6448-6454

10. Grun JL, Maurer PH (1989) Different T helper cell subsets elicited in mice utilizing two different adjuvant vehicles: the role of endogenous interleukin 1 in proliferative responses. Cell Immunol 121(1):134-145. https://doi.org/10.1016/0008-8749(89)90011-7

11. Marrack P, McKee AS, Munks MW (2009) Towards an understanding of the adjuvant action of aluminium. Nat Rev Immunol 9(4): 287-293. https://doi.org/10.1038/nri2510

12. Arif S, Leete P, Nguyen V et al (2014) Blood and islet phenotypes indicate immunological heterogeneity in type 1 diabetes. Diabetes 63(11):3835-3845. https://doi.org/10.2337/db14-0365

13. Eugster A, Lindner A, Heninger AK et al (2013) Measuring T cell receptor and $\mathrm{T}$ cell gene expression diversity in antigen-responsive human $\mathrm{CD}^{+}{ }^{+} \mathrm{T}$ cells. J Immunol Methods 400-401:13-22. https:// doi.org/10.1016/j.jim.2013.11.003

14. Eugster A, Lindner A, Catani M et al (2015) High diversity in the TCR repertoire of GAD65 autoantigen-specific human $\mathrm{CD}^{+} \mathrm{T}$ cells. J Immunol 194(6):2531-2538. https://doi.org/10.4049/ jimmunol.1403031

15. Lefranc MP, Giudicelli V, Ginestoux $C$ et al (2009) IMGT, the international ImMunoGeneTics information system. Nucleic Acids Res 37(Database issue):D1006-D1012. https://doi.org/10. 1093/nar/gkn838

16. Berthold MR, Cebron N, Dill F et al (2008) KNIME: The Konstanz Information Miner. Stud Class Data Anal 11(1):319-326. https:// doi.org/10.1145/1656274.1656280

17. Nazarov VI, Pogorelyy MV, Komech EA et al (2015) tcR: an R package for $\mathrm{T}$ cell receptor repertoire advanced data analysis. BMC Bioinformatics 16(1):175. https://doi.org/10.1186/s12859-0150613-1

18. Gomez-Tourino I, Kamra Y, Baptista R, Lorenc A, Peakman M (2017) T cell receptor beta-chains display abnormal shortening and repertoire sharing in type 1 diabetes. Nat Commun 8(1):1792. https://doi.org/10.1038/s41467-017-01925-2

19. Karlsson Faresjo MG, Ernerudh J, Ludvigsson J (2004) Cytokine profile in children during the first 3 months after the diagnosis of type 1 diabetes. Scand J Immunol 59(5):517-526. https://doi.org/ 10.1111/j.0300-9475.2004.01420.x

20. Arif S, Gibson VB, Nguyen V et al (2017) $\beta$-Cell specific Tlymphocyte response has a distinct inflammatory phenotype in children with type 1 diabetes compared with adults. Diabet Med 34(3): 419-425. https://doi.org/10.1111/dme.13153

21. Britanova OV, Putintseva EV, Shugay M et al (2014) Age-related decrease in TCR repertoire diversity measured with deep and normalized sequence profiling. J Immunol 192(6):2689-2698. https://doi.org/10.4049/jimmunol.1302064

22. Becattini S, Latorre D, Mele F et al (2015) T cell immunity. Functional heterogeneity of human memory $\mathrm{CD}^{+} \mathrm{T}$ cell clones primed by pathogens or vaccines. Science 347(6220):400-406. https://doi.org/10.1126/science.1260668 
23. Tavira B, Barcenilla H, Wahlberg J, Achenbach P, Ludvigsson J, Casas R (2018) Intralymphatic glutamic acid decarboxylase-alum administration induced Th2-like-specific immunomodulation in responder patients: a pilot clinical trial in type 1 diabetes. J Diabetes Res 2018:9391845-9391811. https://doi.org/10.1155/ 2018/9391845

24. Ludvigsson J, Krisky D, Casas R et al (2012) GAD65 antigen therapy in recently diagnosed type 1 diabetes mellitus. N Engl J Med 366(5):433-442. https://doi.org/10.1056/NEJMoa1107096

25. Aly L, Yousef S, Schippling S et al (2011) Central role of JC virusspecific $\mathrm{CD}^{+}$lymphocytes in progressive multi-focal leucoencephalopathy-immune reconstitution inflammatory syndrome. Brain 134(Pt 9):2687-2702. https://doi.org/10.1093/ brain/awr206

26. Globig AM, Hennecke N, Martin B et al (2014) Comprehensive intestinal $\mathrm{T}$ helper cell profiling reveals specific accumulation of IFN- $\gamma^{+} \mathrm{IL}-17^{+}$coproducing $\mathrm{CD} 4^{+} \mathrm{T}$ cells in active inflammatory bowel disease. Inflamm Bowel Dis 20(12):2321-2329. https://doi. org/10.1097/MIB.0000000000000210

27. Kluger MA, Nosko A, Ramcke T et al (2017) ROR $\gamma t$ expression in Tregs promotes systemic lupus erythematosus via IL-17 secretion, alteration of Treg phenotype and suppression of Th2 responses. Clin Exp Immunol 188(1):63-78. https://doi.org/10.1111/cei.12905
28. Hegazy AN, Peine M, Helmstetter $\mathrm{C}$ et al (2010) Interferons direct Th2 cell reprogramming to generate a stable GATA- $3^{+} \mathrm{T}^{-}$bet ${ }^{+}$cell subset with combined $\mathrm{Th} 2$ and $\mathrm{Th} 1$ cell functions. Immunity $32(1)$ : 116-128. https://doi.org/10.1016/j.immuni.2009.12.004

29. Axelsson S, Cheramy M, Hjorth M et al (2011) Long-lasting immune responses 4 years after GAD-alum treatment in children with type 1 diabetes. PLoS One 6(12):e29008. https://doi.org/10. 1371/journal.pone.0029008

30. Peine M, Rausch S, Helmstetter C et al (2013) Stable T-bet ${ }^{+}$GATA$3^{+}$Th1/Th2 hybrid cells arise in vivo, can develop directly from naive precursors, and limit immunopathologic inflammation. PLoS Biol 11(8):e1001633. https://doi.org/10.1371/journal.pbio. 1001633

31. Harrison OJ, Linehan JL, Shih HY et al (2019) Commensal-specific $\mathrm{T}$ cell plasticity promotes rapid tissue adaptation to injury. Science 363(6422):eaat6280. https://doi.org/10.1126/science.aat6280

32. Pihl M, Barcenilla H, Axelsson S et al (2017) GAD-specific T cells are induced by GAD-alum treatment in type-1 diabetes patients. Clin Immunol 176:114-121. https://doi.org/10.1016/j.clim.2017. 01.010

Publisher's note Springer Nature remains neutral with regard to jurisdictional claims in published maps and institutional affiliations. 